\title{
RESEARCH HIGHLIGHT Reprogramming the microenvironment: tricks of tumor-derived astrocytes
}

\author{
Guoxin Zhang ${ }^{1}$ and Jeremy N. Rich (D) ${ }^{1}$ \\ Cell Research (2020) 30:633-634; https://doi.org/10.1038/s41422-020-0335-4
}

\begin{abstract}
Medulloblastomas generate a complex ecosystem through the simultaneous evolution of tumor progenitor cells and transdifferentiated progeny (tumor-derived astrocytes). In a recent Cell paper, Yao et al. show that tumor-derived astrocytes secrete interleukin-4 to reprogram the innate immune system by polarizing resident brain microglia towards protumorigenic tumor-associated macrophages that secrete insulin-like growth factor 1 , feeding back to stimulate tumor progenitor proliferation.
\end{abstract}

Cancers coopt aberrant developmental processes and wound responses ("the wound that does not heal"). Collectively, tumor initiation and growth are driven by dysregulation of cellular differentiation and the tumor microenvironment. Pathologists classify and grade tumors informed by histologic features that often include cellular morphology, cellular markers reflective of differentiation state and identity, and stromal elements (including vasculature, extracellular matrix, and inflammatory cells). Nomenclature of tumors integrates tissue of origin and cell type, but cancers often display multiple cell types or aberrant marker expression. These efforts assume that cancers adhere to the normal sequence and rules of differentiation during initiation and growth, despite extensive evidence of extensive cellular plasticity in tumors. Indeed, the molecular regulators of induced pluripotency and directed differentiation are dysregulated in cancer. Based on this background, cancer biologists have begun to investigate the interplay between cellular differentiation state and the tumor microenvironment. In brain tumors, multiple groups have identified transdifferentiation of glioblastoma stem-like cells into endothelial cells, potentially contributing to resistance to anti-angiogenic therapy. ${ }^{1,2}$ The lineage plasticity in glioblastoma was further demonstrated by tumor-derived pericytes, ${ }^{3}$ which may serve as a reservoir for tumor recurrence and represent a viable cellular target to augment the efficacy of chemotherapy. ${ }^{4}$

Medulloblastoma is the most common primary malignant childhood brain tumor. As a pediatric cancer, medulloblastoma displays striking connections to developmental pathways with classification including sonic hedgehog (SHH)-, Wnt-, and Myc-driven tumors, among others. Genetically engineered models (GEMs) of medulloblastoma have empowered the investigation of tumor initiation. While several genetic lesions have been used to generate medulloblastomas, frequently $\mathrm{SHH}$-driven medulloblastomas have been a focus, based on deletion of Ptch1, the cell surface receptor for Shh. ${ }^{5}$ In a recent issue of Cell, Yao and colleagues leveraged Mosaic Analysis with Double Markers (MADM) to perform lineage tracing studies in a SHH medulloblastoma GEM. ${ }^{6}$ MADM employs Cre/loxpinduced interchromosomal mitotic recombination to generate
GFP-labeled knockout cells and RFP-labeled wild-type cells from colorless heterozygous cells in somatic tissues (Fig. 1a). Under conventional Cre/loxp GEMs, the reporter and the gene of interest reside on separate loci, requiring independent events to activate the reporter and delete the target gene. In contrast, MADM genetically links the reporter and target gene, augmenting the reliability of the reporter as connected to genetic modifications and improving lineage tracing. The dependency of MADM on Cre/loxp-induced interchromosomal mitotic recombination reduces the efficiency of genetic targeting, potentially mimicking the clonal origin of cancer in human patients and providing single-cell resolution for lineage tracing.

In the new MADM-based genetic model, a Ptch1-heterozygous, p53-heterozygous mouse in which the remaining $p 53$ allele was targeted in granule neuron progenitors (GNPs) using a Math1-Cre driver generated medulloblastomas with GFP-labeled tumor GNPs (TuGNPs). Surprisingly, astrocytes within the tumor were also GFP positive, supporting a tumor origin, despite comprising part of the tumor microenvironment. Transdifferentiation of TuGNPs to an astrocytic lineage (tumor-derived astrocytes, TuAstrocytes) suggests that the differentiation within medulloblastomas does not follow the canonical differentiation cascade in normal development, as GNPs would not be expected to yield astrocytes. This observation was not a laboratory artifact of a GEM, as human medulloblastoma samples interrogated for loss of PTCH1 by fluorescent in situ hybridization, showed that tumor cells and astrocytes, identified by immunofluorescent staining for the GFAP astrocyte marker, shared the same karyotype, confirming that astrocytes and tumor cells are lineage related in patients.

To address the potential functional significance of lineage plasticity in tumors, the authors quantified TuAstrocytes relative to proliferation of TuGNPs in preneoplastic lesions, revealing a strong positive correlation. Further, in vitro culture experiments demonstrated that TuAstrocytes accelerated the growth of tumor cells, suggesting a supportive role of transdifferentiated stromal elements in tumor cell proliferation. While these data suggest that TuAstrocytes have a direct paracrine effect on TuGNPs, the tumor yielded more complexity. TuAstrocytes promoted tumor growth through immune modulation by secreting interleukin-4 (IL4) to induce polarization of microglia into tumor-associated macrophages (TAMs), which in turn promoted tumor growth through the secretion of insulin-like growth factor 1 (IGF1) (Fig. 1b). The functional importance of IGF1 signaling in this process was supported by genetic targeting of the IGF1 receptor (IGF1R) in SHH medulloblastoma GEM or blocking of IGF1 signaling with IGFBP3 in vitro. The genetic models also resolved an origin question of TAMs in these tumors, with

${ }^{1}$ Division of Regenerative Medicine, Department of Medicine, University of California, San Diego, CA 92037, USA

Correspondence: Jeremy N. Rich (drjeremyrich@gmail.com)

Published online: 18 May 2020 
a

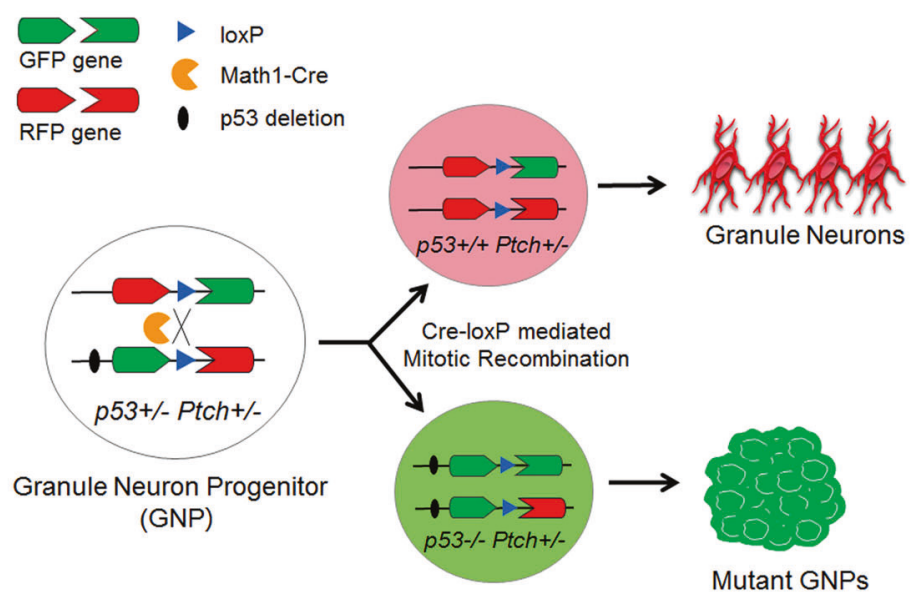

b
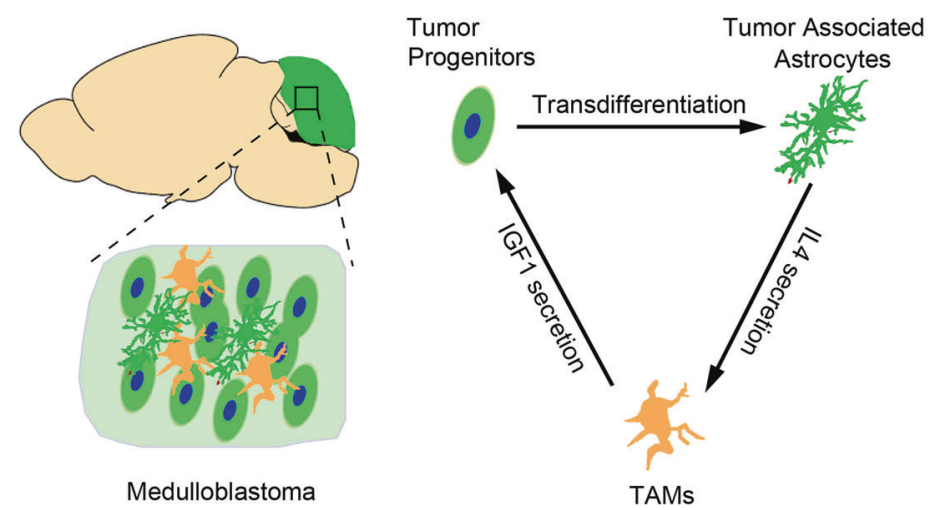

Fig. 1 MADM-based medulloblastoma modeling and the complex ecosystem in medulloblastoma through the simultaneous evolution of tumor progenitor cells and transdifferentiated progeny (tumor-derived astrocytes). a The $p 53$ null allele is linked to GFP in a Ptch1heterozygous, p53-heterozygous mouse. Upon Math1-Cre mediated mitotic recombination, p53 null, Ptch1-heterozygous tumor cells will be labeled with GFP to empower lineage tracing of progeny. b Tumor-derived astrocytes secrete IL4 to reprogram the innate immune system by polarizing resident brain microglia towards pro-tumorigenic TAMs that secrete IGF1, feeding back to stimulate tumor progenitor proliferation.

resident microglia or circulating monocytes as both potential sources, but lineage tracing firmly established the microglia as the origin of these TAMs. Collectively, these studies suggest that even a relatively simple genetic cancer generates tumors with a complex interplay between the tumor microenvironment and the cell of origin.

This exciting study highlights the importance of transdifferentiation of tumor cells in $\mathrm{SHH}$-driven medulloblastoma and opens up doors for new investigation. First, direct interrogation of the functional importance of TuAstrocytes to tumor growth can be supported in vivo by targeting them genetically or pharmacologically. Of further interest, it will be exciting to determine how prevalent these findings are in other subtypes of medulloblastoma and other brain tumors to promote tumor growth. Finally, the cues that lead to aberrant differentiation of GNPs into astrocytes will be important, as these processes may also be amenable to therapeutic targeting. For example, the transdifferentiation of glioblastoma stem-like cells into pericytes involves an SDF1/CXCR4 axis to recruit tumor cells to the vasculature where they are induced to assume a pericytic lineage through TGF $\beta$ signaling. ${ }^{3}$

Tumor recruitment of vasculature and reprogramming of the immune system is a firmly established fact that has been exploited to generate new weapons in oncology through targeted agents, like anti-angiogenics and immune checkpoint inhibitors. However, these therapies are effective only in subsets of cancers and usually are not curative. The current studies demonstrate that therapeutic paradigms based on simple linear relationships between tumor cells and the tumor microenvironment demand greater depth of study. Tumor cells are not simply the recipient of microenvironmental cues, rather they serve as architects of their environment through not only paracrine factors but also through the generation of microenvironmental cells through transdifferentiation. While tumor precursors are often highlighted as the essential cells driving tumor growth, differentiated progeny in other brain tumors (glioblastoma) provide critical feedback cues to the precursors to promote tumor growth. ${ }^{7}$ Thus, dysregulated tumor progeny may offer additional therapeutic targets that alone or in combination with conventional or newer therapies could improve the treatment of cancer patients.

\section{ADDITIONAL INFORMATION}

Competing interests: The authors declare no competing interests..

\section{REFERENCES}

1. Wang, R. et al. Nature 468, 829-833 (2010).

2. Soda, Y. et al. Proc. Natl. Acad. Sci. USA 108, 4274-4280 (2011).

3. Cheng, L. et al. Cell 153, 139-152 (2013).

4. Zhou, W. et al. Cell Stem Cell 21, 591-603 (2017).

5. Goodrich, L. V., Milenković, L., Higgins, K. M. \& Scott, M. P. Science 277, 1109-1113 (1997).

6. Yao, M. et al. Cell 180, 502-520 (2020).

7. Wang, X. et al. Cell Stem Cell 22, 514-528 (2018). 